\title{
A Sliding Observer for Robust Flux Estimation of Induction Motors and Its Characteristics
}

\author{
Somboon Sangwongwanich*, Tsuyoshi Yonemoto*, \\ Takeshi Furuhashi* and Shigeru OKumA*
}

\begin{abstract}
This paper proposes a method of estimating the rotor flux using a sliding observer together with a quantitative design method of robust observer based on disturbance rejection capability in time domain. The proposed design method is valid also for the reduced-order observer, hence yields a solution to the problem of designing a robust reduced-order observer which is not clearly understood at present. It is clarified that the designed sliding observer has several advantages over the reduced-order observer. The dynamic and steady-state characteristics of the estimation error are shown to be determined by $H_{\infty}$-norm of the transfer function of the disturbances.
\end{abstract}

Key Words: sliding observer, induction motor, robustness, ultimate boundedness

\section{Introduction}

Since the advent of the field-oriented control of squirrel-cage induction motors (IM's) by Blasche and Hasse based on physical insight of the machine behaviors, lots of attempts have been made to find out new control strategies which are more theoretical and/or robust than the field-oriented control. Such control strategies are mainly developed on the assumption that the exact value of rotor flux is known in advance. As the rotor flux cannot be directly measured, numerous researches have been carried out to estimate the rotor flux using various kinds of observers. Among the observers investigated so far, the reduced-order observer is quite popular due to its simple pole assignment and low dimension property. It is well-known that the sensitivity of the reduced-order observer to the dis-

\footnotetext{
* Faculty of Engineering, Nagoya University,

Furoh-cho, Chikusa-ku, Nagoya

(Received August 20, 1990)

(Revised February 12, 1991)
}

turbances is high in the low speed region due to a large feedback gain corresponding to a fixed pole assignment. Because of lack of both proper quantitative analysis on the observer performances and development of robust design against disturbances, the observers often suffer from measurement noises and parameter deviations. As the characteristics of the controllers are dominated by the error in the flux estimation, it is of importance to evaluate the error of the observer caused by the disturbances.

In Ref. 8), a method of estimating the rotor flux using a sliding observer together with a quantitative design method of a robust observer in the frequency domain has been developed based on disturbance rejection capability and convergence rate of estimation error. In this paper, the characteristics of the proposed sliding observer in the time domain is clarified, and the boundedness of the estimation error is analyzed. The quantitative analysis presented makes it possible to evaluate the estimation error, and hence gives the guidelines on pole assignment for both the sliding and the reduced-order observers. In addition, it is shown that the reduced-order observer can only be designed with a fixed (time-invariant) feedback gain, therefore giving a narrower class of observers than does the sliding observer. Finally, the validity of the proposed design method is verified by simulation.

\section{Construction of Sliding Observer and Its Characteristics}

\subsection{Characteristics of Sliding Observer on Sliding Hyperplane}

Consider a linear time-varying system expressed in Gopinath's form as in Eqs. (1)-(3). 


$$
\begin{aligned}
& \dot{x}_{1}=A_{11}(t) x_{1}+A_{12}(t) x_{2}+B_{1}(t) u \\
& \dot{x}_{2}=A_{21}(t) x_{1}+A_{22}(t) x_{2}+B_{2}(t) u \\
& y=C x=\left[\begin{array}{ll}
I & 0
\end{array}\right] x=x_{1} .
\end{aligned}
$$

Hereafter the variable $t$ denoting the time-varying terms is omitted. A sliding observer for the above system can be constructed as

$$
\begin{aligned}
& \dot{\hat{x}}_{1}=A_{11} \hat{x}_{1}+A_{12} \hat{x}_{2}+B_{1} u+K_{1} \operatorname{sgn}(\hat{y}-y) \\
& \dot{\hat{x}}_{2}=A_{21} \hat{x}_{1}+A_{22} \hat{x}_{2}+B_{2} u-L K_{1} \operatorname{sgn}(\hat{y}-y) \\
& \hat{y}=C \hat{x}=\left[\begin{array}{ll}
I & 0
\end{array}\right] \hat{x}=\hat{x}_{1}
\end{aligned}
$$

with the sliding hyperplane defined by

$$
\xi=\hat{y}-y=0 \text {. }
$$

where $\wedge$ denotes the estimated value. Then, the error equations become

$$
\begin{aligned}
& \dot{e}_{1}=\dot{\hat{x}}_{1}-\dot{x}_{1}=A_{11} e_{1}+A_{12} e_{2}+K_{1} \operatorname{sgn}(\hat{y}-y) \quad(8) \\
& \dot{e}_{2}=\dot{\hat{x}}_{2}-\dot{x}_{2}=A_{21} e_{1}+A_{22} e_{2}-L K_{1} \operatorname{sgn}(\hat{y}-y)
\end{aligned}
$$

A sliding mode occurs if the gain $K_{1}$ is chosen large enough. Let us assume that the sliding mode does occur. Using the equivalent control method ${ }^{6)}$.

$$
\xi=\hat{y}-y=0, \quad \dot{\xi}=\dot{\hat{y}}-\dot{y}=0
$$

then the error equation on the sliding hyperplane becomes

$$
\dot{e}_{2}=\left(A_{22}+L A_{12}\right) e_{2} .
$$

In comparison, the reduced-order observer is constructed by feedback of the error of the differential of the output as

$$
\begin{aligned}
\dot{z}= & \left(A_{21}+L A_{11}\right) x_{1}+\left(A_{22}+L A_{12}\right)(z-L y) \\
& +\left(B_{2}+L B_{1}\right) u \\
\tilde{x}_{2}= & z-L y
\end{aligned}
$$

where also denotes the estimated value.

The variable $z$ is introduced to avoid differential operation. The error equation is obtained as

$$
\dot{\hat{e}}_{2}=\dot{\tilde{x}}_{2}-\dot{x}_{2}=\left(A_{22}+L A_{12}\right) \tilde{e}_{2}-\dot{L} y \text {. }
$$

Hence, the characteristic of the sliding observer on the sliding hyperplane (11) is the same as that of the reduced-order observer (14) if $L$ is held constant. However, the advantage of the sliding observer over the reduced-order observer is that the feedback gain $L$ is allowed to be time-varying. As for the reduced-order observer, the second term on the right-hand side of Eq. (14) enters into the error dynamics as a disturbance, the effect of which is severe when the observer is sensitive to the disturbance.

The similarity just stated implies that the sliding observer possesses internally a differentiator. The reason for the similarity is as follows. Firstly, the expression for the signal $K_{1} \operatorname{sgn}(\hat{y}-y)$ can be obtained from Eq. (8) as

$$
\begin{aligned}
& K_{1} \operatorname{sgn}(\hat{y}-y) \\
& \quad=K_{1} \operatorname{sgn}\left[\int\left(A_{11} e_{1}+A_{12} e_{2}\right) d t+\int K_{1} \operatorname{sgn}(\hat{y}-y) d t\right]
\end{aligned}
$$

The corresponding block diagram is shown in Fig. $\mathbf{1}$ which includes also the case where the measurement output $y$ is contaminated by the noise $\eta$, i. e. $y=x_{1}+\eta$. The portion of the block diagram which is enclosed with the dotted line is, in fact, a kind of variable structure filter $\left.{ }^{10)}, 11\right)$. For the case of no measurement noise $(\eta=0)$, during the sliding mode the transfer function of the variable structure filter $P_{e q}(s)$ becomes $P_{e q}(s)=-s$. So, it is clear that the differentiation is performed by the variable structure filter. Also, in such a case the input to the variable structure filter becomes $\frac{1}{\mathrm{~s}}$ $\left[A_{12} e_{2}\right]$, because $e_{1}=0$. As a consequence, the output of the variable structure filter $K_{1} \operatorname{sgn}(\hat{y}-y)$ is equivalent to $-A_{12} e_{2}$, and this value is fed back through the gain $L$ to the error equation of $e_{2}$. This is the principal reason why the sliding observer behaves like the reduced-order observer. On the other hand, the difference in the way of obtaining the differential signal yields a different characteristic against the measurement noises. Though it is not shown here in details, considering that the equivalent gain of the switching element depends on the input variable, the equivalent transfer function of the variable structure filter when the measurement noise $\eta=\left[\eta_{i}\right]^{T}$ exists, is given $b^{8)}$

$$
\begin{aligned}
& P_{e q}(s)=\left[s I-K_{e q}\right]^{-1} s K_{e q} \\
& K_{e q}=\operatorname{diag}\left(\left[k_{e q}\right]_{i}\right), \quad\left[k_{e q}\right]_{i} \propto 1 / \eta_{i} .
\end{aligned}
$$

Hence, the variable structure filter now is not purely the differentiator but incorporates also a low. pass filter whose cut-off frequency varies with the

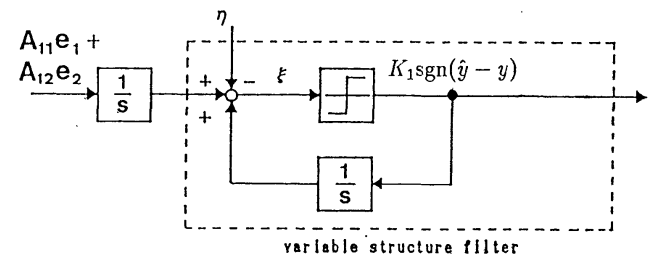

Fig. 1 Interpretation of error equation of $e_{1}$ using variable structure filter 


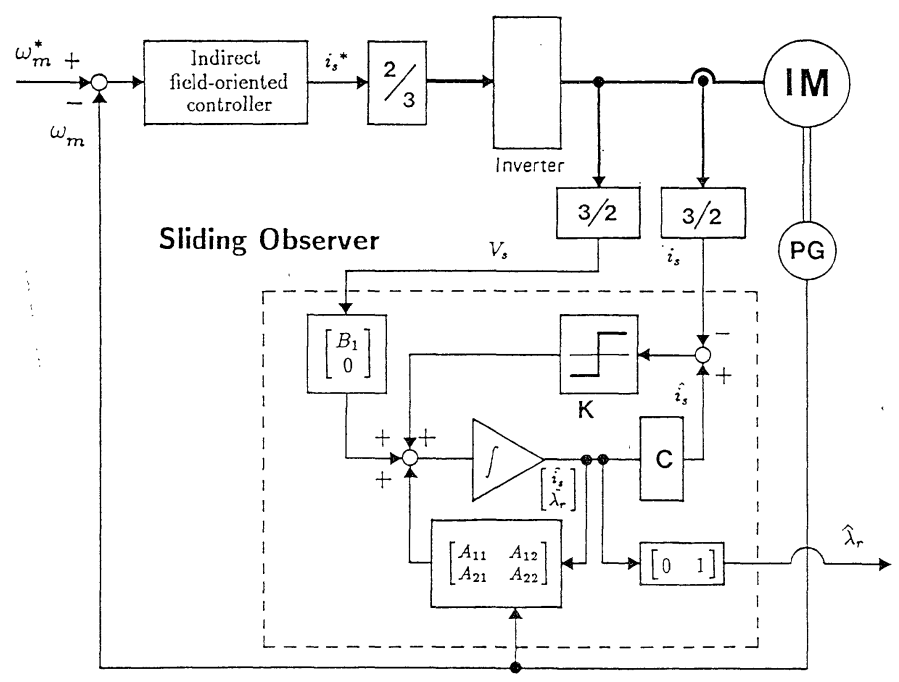

Fig. 2 Sliding observer for induction machines

measurement noise. This result also clarifies that the sliding observer possesses a kind of adaptive filtering capability against measurement noises ${ }^{7)}$, 10), 11).

In the sliding observer, the sliding hyperplane is determined by the system itself whereas the switching gain $K$ can be arbitrary assigned to attain robustness against disturbances which satisfy

$$
\operatorname{Im} D \subset \operatorname{Im}\left[\begin{array}{c}
K_{1} \\
-L K_{1}
\end{array}\right]=\operatorname{Im} K
$$

where $D$ denotes the input matrix of the disturbances. However, the restriction on the assignment of the switching gain $K$ arising from the stability requirement is

$$
\begin{aligned}
& \text { transmission zeros of } C(s I-A)^{-1} K \\
& \quad \in \text { open left half-plane. }
\end{aligned}
$$

Therefore, a trade-off must be considered between the robustness and the stability.

\subsection{Construction of Sliding Observer for Induction Motors}

In this section, construction of a sliding observer for estimation of rotor flux of an induction motor is described. The state equations of the induction motor in rectangular $(d-q)$ coordinates fixed to the stator are given by

$$
\frac{d}{d t}\left[\begin{array}{l}
i_{s} \\
\lambda_{r}
\end{array}\right]=\left[\begin{array}{ll}
A_{11} & A_{12} \\
A_{21} & A_{22}
\end{array}\right]\left[\begin{array}{l}
i_{s} \\
\lambda_{r}
\end{array}\right]+\left[\begin{array}{c}
B_{1} \\
0
\end{array}\right] v_{s}+D w
$$

where

$w:$ disturbance vector

$$
\begin{aligned}
& A_{11}=-\left(R_{s}+R_{r} M^{2} / L_{r}^{2}\right) /\left(\sigma L_{s}\right) \cdot I \\
& A_{12}=\left(R_{r} M / \sigma L_{s} L_{r}^{2}\right) \cdot I-P \omega_{m} M /\left(\sigma L_{s} L_{r}\right) \cdot J \\
& A_{21}=\left(M R_{r} / L_{r}\right) \cdot I \\
& A_{22}=-\left(R_{r} / L_{r}\right) \cdot I+P \omega_{m} \cdot J \\
& B_{1}=\left(1 / \sigma L_{s}\right) \cdot I, \quad \sigma=1-M^{2} /\left(L_{s} L_{r}\right) \\
& I=\left[\begin{array}{ll}
1 & 0 \\
0 & 1
\end{array}\right], \quad J=\left[\begin{array}{cc}
0 & -1 \\
1 & 0
\end{array}\right] .
\end{aligned}
$$

The state variables are the stator current $i_{s}$ and the rotor flux $\lambda_{r}$. The measurable output is $i_{s}$. The control input is the stator voltage $v_{s}$. Definitions of the parameters are given in Appendix.

As described in the previous section, the sliding observer for the induction motor is constructed as

$$
\begin{aligned}
& \hat{i}_{s}=A_{11} \hat{i}_{s}+A_{12} \hat{\lambda}_{r}+B_{1} v_{s}+K_{1} \operatorname{sgn}\left(\hat{i}_{s}-i_{s}\right) \\
& \hat{\lambda}_{r}=A_{21} \hat{i}_{s}+A_{22} \hat{\lambda}_{r}-L K_{1} \operatorname{sgn}\left(\hat{i}_{s}-i_{s}\right)
\end{aligned}
$$

Fig. 2 shows the configuration of the sliding observer. The sufficient conditions that guarantee the existence of the sliding mode are given in Ref. 8).

\subsection{Geometrical Structures of Parameter Deviations}

The vector $w$ and the input matrix $D$ in Eq. (19) referring to the disturbance vector and the geometrical structure of the disturbance, respectively, are useful in evaluating quantitatively the estimation errors of the rotor flux derived in the sequel. From the state equations (19), the disturbance input matrix $D$ and the disturbance vector $w$ for some parameter deviations are obtained as shown 
below :

$$
\text { ( a ) } \begin{aligned}
& R_{s} \rightarrow R_{s}+\Delta R_{s}: \\
D= & {[I 0]^{T}, \quad w=\left(\Delta R_{s} / \sigma L_{s}\right) i_{s} } \\
\text { (b) } \cdot & R_{r} \rightarrow R_{r}+\Delta R_{r}: \\
D= & {[I-\varepsilon I]^{T}, \quad w=\left(\Delta R_{r} M / \varepsilon L_{r}\right) i_{T} } \\
i_{T}= & i_{s}-\lambda_{r} / M: \text { torque current }
\end{aligned}
$$

In the case of multiple disturbances, $D$ and $w$ can be modified and the disturbances can be treated in the same manner.

\section{Design of Robust Sliding Observer against Disturbances}

Firstly, we will make a brief review of the design method in the frequency domain derived in Ref. 8). For the design of a robust sliding observer against disturbances, effects of the disturbance Dw on the characteristics of the observer on the sliding hyperplane must be clarified. Input matrix $F$ of the disturbance in the error equation on the sliding hyperplane is derived by using the equivalent control method as

$$
F=\left[\begin{array}{ll}
L & I
\end{array}\right] D .
$$

Hence, the error equation of the observer on the sliding hyperplane becomes

$$
\dot{e}_{2}=\bar{A} e_{2}+F w
$$

where

$$
\begin{aligned}
& \bar{A}=A_{22}+L A_{12}=-\alpha I+\beta J \quad(\alpha>0) \\
& L=\left[\varepsilon-\frac{c \alpha+d \beta}{c^{2}+d^{2}}\right] I+\left[\frac{-d \alpha+c \beta}{c^{2}+d^{2}}\right] J \\
& c=R_{r} / L_{r} \varepsilon, \quad d=P \omega_{m} / \varepsilon \\
& \varepsilon=\sigma L_{s} L_{r} / M .
\end{aligned}
$$

The design parameters are, thus, the poles of the closed-loop error system $-\alpha \pm j \beta$ or equivalently the feedback matrix $L$.

It should be noted that the error system just stated is the same as that of the reduced-order observer, therefore the design method developed in the following is valid also for the reduced-order observer.

\section{(1) Disturbance Rejection}

Let the transfer function from the disturbance $w$ to the estimation error $e_{2}$ be denoted by $T(s)$. The effects of the disturbance to the estimation error can be evaluated using a suitable norm of $T(s)$. It is natural to adopt $H_{\infty}$-norm of the transfer function $\|T\|_{\infty}$ for the worst case design, because the frequency spectrum of $w$ is not known. As $\|T\|_{\infty}$ is the ratio of the estimation error energy to the disturbance energy in the worst case, this means that we evaluate the characteristic of the error system in a sense of integral of the squared error. From Eq. (23), $\|T\|_{\infty}$ is obtained as

$$
\|T\|_{\infty}=\sup _{\omega} \sigma_{\max }[T(j \omega)]=\|F\| / \alpha
$$

where

$$
T(s)=(s I-\bar{A})^{-1} F .
$$

$\sigma_{\max }[\cdot]$ denotes the maximum singular value and $\|\cdot\|$ denotes the induced norm.

It is evident that in order to reduce the sensitivity of the observer to disturbances, $\|F\|$ should be designed small or the real part of the pole of the closed-loop system $\alpha$ should be made large. However, $\|F\|$ and $\alpha$ cannot be adjusted independently.

(2) Convergence Rate of Estimation Error

Besides the low sensitivity against disturbances, the initial error should be quickly attenuated. Evaluation of the attenuation characteristics can be achieved using $\mathrm{H}_{2}$-norm of the transfer function of the closed-loop system. $H_{2}$-norm of the transfer function $\|G\|_{2}$ is defined by

$$
\begin{aligned}
\|G\|_{2}^{2} & =(1 / 2 \pi) \int_{-\infty}^{\infty} \operatorname{trace} G(-j \omega)^{T} G(j \omega) d \omega \\
& =1 / \alpha
\end{aligned}
$$

where

$$
G(s)=(s I-\bar{A})^{-1} .
$$

$G(s)$ is the closed-loop transfer function of the error system. Physically, $\|G\|_{2}$ is the mean-square value of the estimation error corresponding to a stochastic noise and is also equal to the integral of the square of the impulse response of the error system to the initial error. Therefore, to improve the convergence rate of the estimation error, the real part of the poles of the closed-loop transfer function $\alpha$ should be made large.

Normally, $\|T\|_{\infty}$ is large for large $\alpha$ which means that the disturbance rejection capability and the convergence rate of the estimation error should be traded off.

\subsection{Frequency Domain Design}

The design method of a robust observer proposed in Ref. 8) basically attempts to obtain fast 
convergence of the estimation error, while the disturbance attenuation capability is constrained by a prescribed value, i.e.,

$\|G\|_{2} \rightarrow \min$ with $\|T\|_{\infty} \leq \gamma$

This optimization problem involves, in fact, only solving an algebraic equation, and therefore can be solved analytically (Fig. 3). Concrete design examples are shown in the following:

(A1) $D=\left[\begin{array}{ll}I & 0\end{array}\right]^{T}$ : The optimal solutions are

$$
\begin{aligned}
& \alpha^{*}=\frac{R_{r} / L_{r}}{1-\gamma \sqrt{c^{2}+d^{2}}}, \quad \beta^{*}=p \omega_{m} \\
& L^{*}=-\left(\alpha^{*}-R_{r} / L_{r}\right)\left\{\left[\frac{c}{c^{2}+d^{2}}\right] I+\left[\frac{d}{c^{2}+d^{2}}\right] J\right\} \\
& \|T\|_{\infty}^{*}=\frac{\left|\alpha^{*}-R_{r} / L_{r}\right|}{\alpha^{*} \sqrt{c^{2}+d^{2}}}=\gamma .
\end{aligned}
$$

where * denotes the optimal value.

(A2) $D=[I-\varepsilon I]^{T}$ : In this case, $\alpha$ can be chosen arbitrarily large without violating the condition on $\|T\|_{\infty}$. However, it tends to give dynamically an undesirable large error. Therefore, we introduce the following limit on $\|F\|$ which corresponds to the magnitude of disturbances in the dynamic error equations and is related to the maximum value of the estimation error:

$$
\|F\| \leq \nu \text {. }
$$

Then,

$$
\begin{aligned}
& \alpha^{*}=\nu \sqrt{c^{2}+d^{2}}, \quad \beta=0 \\
& L^{*}=\left[\varepsilon-\frac{c \alpha^{*}}{c^{2}+d^{2}}\right] I-\left[\frac{d \alpha^{*}}{c^{2}+d^{2}}\right] J
\end{aligned}
$$

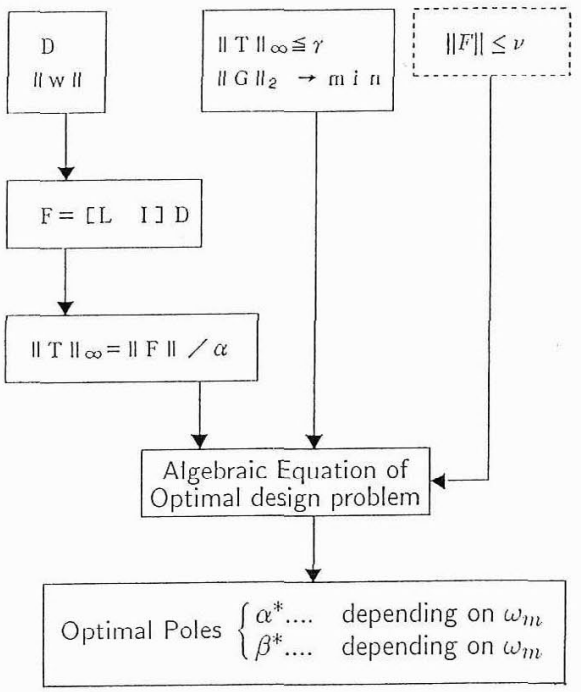

Fig. 3 Algorithm of optimal design method

$$
\|T\|_{\infty}^{*}=1 / \sqrt{c^{2}+d^{2}}
$$

are obtained.

\subsection{Time Domain Design}

As derived in the previous section, to obtain the robust design of the sliding observer, the poles of the closed-loop system $(-\alpha \pm j \beta)$ are assigned to be time-varying depending on the rotor speed $\omega_{m}$. Hence, the stability of the observer is not guaranteed even if the real part of the poles is always negative. Furthermore, in the case where the disturbance exists, it is not sure that the design method in the frequency domain will give a good response in the time domain. This is because the evaluation based on $H_{\mathrm{so}}$-norm corresponds only to the behavior of the observer in the integral sense. Also, bound of the estimation error should be known to designers when the observer is incorporated into the controller. To deal with the problem, we define the following Lyapunov function

$$
V=\frac{1}{2} e_{2}^{T} e_{2} .
$$

Using Eq. (23), the derivative of the Lyapunov function $\dot{V}$ is calculated as

$$
\begin{aligned}
\dot{V} & =e_{2}^{T} \dot{e}_{2}=e_{2}^{T}\left[(-\alpha I+\beta J) e_{2}+F w\right] \\
& =-\alpha\left\|e_{2}\right\|^{2}+e_{2}^{T} F w \\
& \leq\left\|e_{2}\right\| \cdot\left(-\alpha\left\|e_{2}\right\|+\|F\| \cdot \rho\right) \\
& \leq-\Gamma\left(\left\|e_{2}\right\|\right)
\end{aligned}
$$

where

$$
\begin{aligned}
& \Gamma\left(\left\|e_{2}\right\|\right)=\alpha\left\|e_{2}\right\| \cdot\left(\left\|e_{2}\right\|-R_{\max }\right) \\
& R_{\max }=\|T\|_{\infty} \cdot \rho \\
& \rho=\text { bound of }\|w\| .
\end{aligned}
$$

Equations (39) and (41) are depicted in Fig. 4. From the figure, the practical stability (See Ref. 12) for detailed definitions) of the observer is obtained as follows :

[1] Uniform boundechess : $R$ given

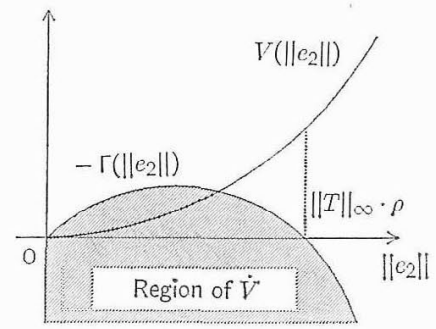

Fig. 4 Lyapunov function and bound of its derivative 
$\left\|e_{2}\left(t_{0}\right)\right\| \leq R \rightarrow\left\|e_{2}(t)\right\| \leq d(R) \quad \forall t \geq t_{0}$

$d(R)= \begin{cases}R & \text { if } R \geq R_{\max } \\ R_{\max } & \text { otherwise. }\end{cases}$

[2] Uniform ultimate boundedness : $R_{0}, R$ given

$$
\begin{aligned}
\left\|e_{2}\left(t_{0}\right)\right\| \leq R_{0} & \\
& \rightarrow\left\|e_{2}(t)\right\| \leq R \quad \text { for } R>R_{\max } t \geq t_{0}+t_{d} \\
t_{d} & = \begin{cases}\left(R_{0}^{2}-R^{2}\right) / 2 \Gamma(R) & \text { if } R_{0}>R ; \\
0 & \text { otherwise. }\end{cases}
\end{aligned}
$$

[3] Uniform stability : $d$ given

$$
\begin{aligned}
& \left\|e_{2}\left(t_{0}\right)\right\| \leq{ }^{\exists} \delta(d)=R_{\max } \\
& \quad \rightarrow\left\|e_{2}(t)\right\| \leq d \quad \forall d>R_{\max } \forall t>t_{0}
\end{aligned}
$$

The sufficient condition for the practical stability is

$$
\alpha(t)>0
$$

which means that the closed-loop poles are allowed to be time-varying. The uniform ultimate boundedness (45) guarantees that the estimation error decreases to $R_{\max }$. Thus, $R_{\max }$ can be regarded as the bound on the amplitude error in steady state. Also, the bound on the phase error denoted by $\theta_{\max }$ in the steady state is simply given by

$$
\theta_{\max }=\tan ^{-1}\left(R_{\max } /\left\|\lambda_{r}\right\|\right)
$$

as depicted in Fig. 5. From Eq. (42), it is apparent that the bound of the estimation error in the steady state is determined by $\|T\|_{\infty}$. The steadystate estimation error caused by parameter deviations in the case of the optimal design proposed are quantitatively evaluated in the following.

In the case of the stator resistance deviation $\left(R_{s} \rightarrow R_{s}+\Delta R_{s}\right)$, using Eqs. (42) and (48), $R_{\max }$ and $\theta_{\max }$ are derived as

$$
\begin{aligned}
& R_{\max }=\frac{\left|\alpha-R_{r}\right| L_{r} \mid}{\alpha \sqrt{c^{2}+d^{2}} \cdot \frac{\left|\Delta R_{s}\right|}{\sigma L_{s}}\left\|i_{s}\right\|} \\
& \theta_{\max }=\tan ^{-1}\left[\frac{\left|\alpha-R_{r} / L_{r}\right|}{\alpha \sqrt{c^{2}+d^{2}}} \cdot \frac{\left|\Delta R_{s}\right|\left\|i_{s}\right\|}{\sigma L_{s}\left\|\lambda_{r}\right\|}\right] .
\end{aligned}
$$

Recalling that from the definition (26) $d$ is proportional to the rotor speed $\omega_{m}$, it is clear from the above equations that $R_{\max }$ and $\theta_{\max }$ vary with $\alpha$

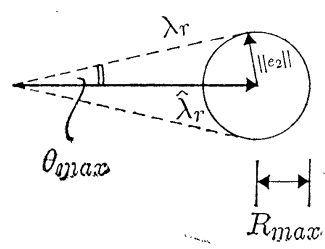

Fig. 5 Ultimate boundedness of sliding observer and the rotor speed $\omega_{m}$. Therefore, to assign $R_{\max }$ and $\theta_{\max }$ to prescribed vaues, $\alpha$ must be changed with the rotor speed $\omega_{m}$.

In the case of the rotor resistance deviation $\left(R_{r} \rightarrow R_{r}+\Delta R_{r}\right), R_{\max }$ and $\theta_{\max }$ are given by

$$
\begin{aligned}
& R_{\max }=\frac{\left|\Delta R_{r} M\right|\left\|i_{T}\right\|}{\varepsilon L_{r} \sqrt{c^{2}+d^{2}}} \\
& \theta_{\max }=\tan ^{-1}\left[\frac{\left|\Delta R_{r} M\right|\left\|i_{T}\right\|}{\varepsilon L_{r} \sqrt{c^{2}+d^{2}}\left\|\lambda_{r}\right\|}\right] .
\end{aligned}
$$

It is clear that $R_{\max }$ and $\theta_{\max }$ are kept minimal regardless of $\alpha$. This reveals the fact that as long as the steady-state estimation error is concerned, $\alpha$ can be set arbitrarily large. However, as stated previously the magnitude of the disturbance term in the error equation depends on $\|F\|$ which increases with $\alpha$, hence a limit on $\|F\|$ should be considered so as not to aggravate the estimation error in the transient state with a large $\alpha$.

From the aforementioned results, it is concluded that the estimation errors of both $R_{\max }$ and $\theta_{\max }$ vary with the rotor speed. The estimation error is small in high speed region and is large in low speed region. Although this is a well-known fact, only in this paper is a quantitative evaluation of the relation between the rotor speed and the estimation error given in an explicit form.

\section{Simulation Results and Discussions}

Simulation is carried out using the block diagram of the control system shown in Fig. 2. Indirect field-oriented control which keeps the rotor flux constant is used. Since the aim of the simulation is to verify the feasibility of the proposed robust design method of the sliding observer, the estimated rotor flux is not fed back to the field-oriented controller for the speed control. The sampling period of the speed controller and the observer are $250(\mu \mathrm{s})$ and $25(\mu \mathrm{s})$, respectively. The sliding observer is realized by discretization of the state equations (20)-(21) using the simple Euler's approximation. A current controller with hysteresis comparators is used in the PWM inverter. The dc voltage of the inverter is $200(\mathrm{~V})$ and the hysteresis bandwidth is 0.5 (A). System constants used in the simulation are listed in Appendix. To see clearly the advantage of the sliding observer de- 
signed, low speed region, where the effects of the disturbances are severe, is mainly simulated.

Simulation is carried out to verify the feasibility of the robust design methods developed. Considering the deviation of the rotor resistance, the pole assignment which minimizes $\|G\|_{2}$ under the constraint of $\|F\| \leq 5.0 \times 10^{-3}$ leads to the poles at $-\alpha \pm$ $j 0$ where $\alpha$ varies with the rotor speed according to Eq. (36) (Fig. 6). Fig. 7 shows the simulation results carried out under the conditions that the demand of the rotor speed $\omega_{m}$ is 100 (rpm), and that at $t=0$, the load torque of $5.0(\mathrm{Nm})$ is applied together with the rotor resistance of the induction motor increased by $50 \%$. In order to investigate the robustness of the observer against the disturbance, the initial estimation error is assumed to be zero. It is clearly seen that the proposed design method gives small estimation error. Moreover, from the results in the time domain design, $R_{\max }=$ 0.062 (Wb) is obtained. And from Fig. 7, it is evident that the estimation error is bounded by the

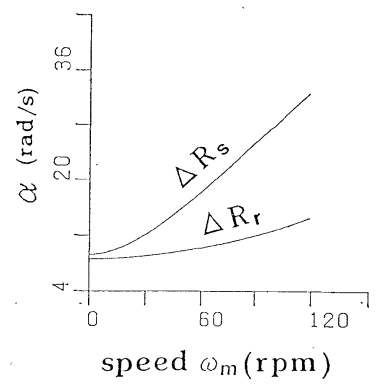

Fig. 6 Optimal pole assignment against parameter deviation
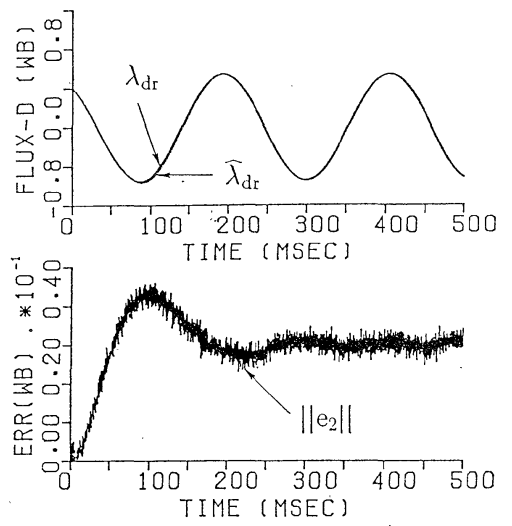

Fig. 7 Simulation results on observer performance against $\Delta R_{r}=50 \%$ with $\|F\|$ given
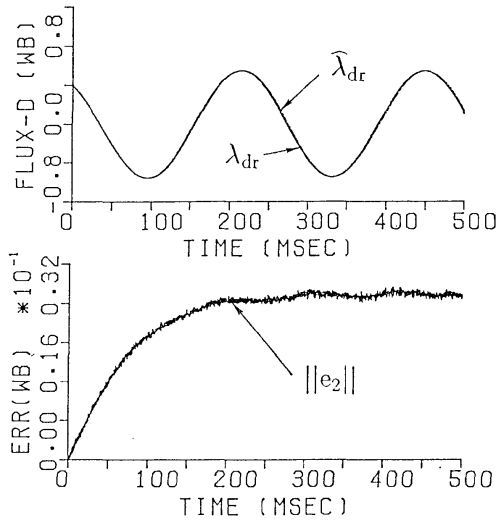

Fig. 8 Simulation results on observer performance against $\Delta R_{s}=50 \%$ with $\|T\|_{\infty}$ given

predicted $R_{\max }$. Similar results are also obtained in the case of the stator resistance deviation. In

Fig. 8, $\|G\|_{2}$ is minimized under the constraint of $\|T\|_{\infty} \leq 1.5 \times 10^{-4}$. The optimal poles are placed at $-\alpha \pm j p \omega_{m}$ where $\alpha$ varies according to Fig. 6. In this case, the predicted $R_{\max }$ is calculated as 0.044 $(\mathrm{Wb})$, and the simulation result clearly satisfies the boundedness condition.

\section{Conclusions}

In this paper, the sliding observer has been proposed for robust estimation of rotor flux of induction motors. It has been clarified that the sliding observer possesses a kind of adaptive filtering capability and can be designed with the time-varying feedback gain which is the principal advantage over the reduced-order observer. The characteristics of the sliding observer in the time domain are studied using the direct method of Lyapunov. As a result, it is shown that the observer with the time-varying poles in the open left half-plane is practically stable even when the disturbances exist. And, it is derived that the estimation error is bounded by a value defined by the product of the size of the disturbance and $\|T\|_{\infty}$ which is the $H_{\infty}$ norm of the transfer function from the disturbance to the estimation error. As a consequence, it is concluded that the design method based on $\|T\|_{\infty}$ yields both good dynamic and steady-state characteristics. In addition, as the estimation error of both the amplitude and the phase can be evaluated, 
it is of benefit when we incorporate the observer with the field-oriented controller, because we can predict the characteristics of the overall controller using the bound of the estimation error. Finally, simulation results also support the theoretical results developed. And the bound on the estimation error computed using $\|T\|_{\infty}$ is obviously not conservative.

The authors would like to express their thanks to Prof. Y. Hosoe of Nagoya University for his valuable advice.

\section{References}

1) G. Verghese and S.R. Sander: Observer for Flux Estimation in Induction Machines, IEEE Trans. Industrial Electronics, IE-35-1, 85/94 (1988)

2) Y. Hori and T. Umeno: Implementation of Robust Flux Observer-Based Field Orientation (FOFO) Controller for Induction Machines, Conf. Record of Ann. Meeting IEEE-IAS, 523/528 (1989)

3) Y. Dote: Stabilization of Controlled Current Induction Motor Drive System via New Nonlinear State Observer, IEEE Trans. Industrial Electronics and Control Instrumentation, IECI-27-2, 77/81 (1980)

4) A. Bellini, et al.: A Bilinear Observer of the State of the Induction Machine, Ricerche di Automatica, 9-1, 70/85 (1978)

5) Berstein, et al.: Stead-State Kalman Filtering with an $H^{\infty}$ Error Bound, System and Control Lett., 12, 9/16 (1989)

6) V. Utkin: Principles of Identification in Sliding Modes, Dokl. Akad. Nauk SSSR, 257-3, 558/561 (1981)
7) J. J. E. Slotine, et al.: On Sliding Observers for Nonlinear Systems, ASME J. of Dynamic Systems, Measurement and Control, 109, 245/252 (1987)

8) S. Sangwongwanich, et al.: Design of Sliding Observer for Estimation of Rotor Flux of Induction Motors, IEE Japan Trans., 110C-4, 279/288 (1990)

9) J. Xu and H. Hashimoto: A VSS Observer for Nonlinear Systems, Proc. of SICE '88, ES 1-4, 777/ 780 (1988)

10) B. Z. Golembo, et al.: Application of PiecewiseContinuous Dynamic Systems to Filtering Problems, Auto. and Remote Cont., 37-3, 369/377 (1976)

11) S. V. Drakunov: An Adaptive Quasioptimal Filter with Discontinuous Parameters, Auto. and Remote Cont., 44-9, 1167/1175 (1983)

12) Y. H. Chen: On the Robustness of Mismatched Uncertain Dynamical Systems, ASME J. of Dynamic Systems, Measurement and Control, 109, 29/35 (1987)

13) H. Watanabe, et al.: A Method of Flux Observer for the Induction Motor, IEE Japan Trans., 109D9, 622/628 (1989)

\section{«ppendix》}

\section{System constants}

$\begin{array}{lll}\text { stator resistance } & R_{s}=0.60 \quad(\Omega) \\ \text { rotor resistance } & R_{r}=0.69(\Omega) \\ \text { stator self-inductance } & L_{s}=0.101(\mathrm{H}) \\ \text { rotor self-inductance } & L_{r}=0.093(\mathrm{H}) \\ \text { mutual inductance } & M=0.093(\mathrm{H}) \\ \text { number of pole pairs } & p=2 \\ \text { maximum stator current } & =18.0 \quad(\mathrm{~A}) \\ \text { dc link voltage } & =200 \quad(\mathrm{~V})\end{array}$

